\section{British Society for Oral \& Maxillofacial Pathology news}

Dr Mary Toner, Consultant Histopathologist, Dublin Dental University Hospital and Associate Professor, Trinity College, Dublin, will take over from Dr Bill Barrett as President of the British Society for Oral \& Maxillofacial Pathology (BSOMP) in April 2021.

The joint conference of the BSOMP and International Association of Oral \& Maxillofacial Pathologists in London 2020 was cancelled. A virtual joint conference in a format more suited to online delivery is being scheduled for June 2021. The Society's annual Undergraduate Essay Prize for 2020, the title of which was 'Should dental students be taught pathology?', has been awarded to Mohammed Al Bux of the University of Sheffield. Further information on the Society, its grants and prizes can be found at the BSOMP website (bsomp.org.uk).

\section{Jason Leitch webinar}

The Faculty of Forensic \& Legal Medicine (FFLM) of the Royal College of Physicians will be hosting a webinar on 25 February 2021 from 6.30-7.30 pm featuring Professor Jason Leitch CBE.

Professor Jason Leitch CBE is currently National Clinical Director in Scotland. He will be speaking on: 'How do you recognise quality in the clinical health field?'

Faculty webinars are open to all and available from the FFLM's website: https://fflm.ac.uk/e-learning/webinars/.

Several dentists and a GDC panel member recently passed the FFLM's Diploma of Legal Medicine. For more information visit www.fflm.ac.uk.

\title{
Coronavirus spread could be reduced with slower drill rotation
}

Researchers at Imperial College London and King's College London have measured and analysed aerosol generation during dental procedures and suggested changes to prevent contamination in the first place, to improve safety for both patients and the dental practice workforce. ${ }^{1}$

They suggest that dentists avoid using dental drills that use a mixture of air and water as the abrasion coolants, and carefully select and control drill rotation speeds for those instruments that only use water as a coolant. Parameters have been identified that would allow some procedures such as dental fillings to be provided while producing 60 times fewer aerosol droplets than conventional instrumentation.

Lead author Dr Antonis Sergis of Imperial's Department of Mechanical Engineering said: 'Aerosols are a known transmission route for the virus behind COVID-19, so, with our colleagues at King's, we have tested suggested solutions that reduce the amount of aerosols produced in the first place. These could help reduce the risk of transmission during dental procedures'.

Co-author Professor Owen Addison of King's College London's Faculty of Dentistry, Oral \& Craniofacial Sciences said: 'This important work describes the basic mechanisms that lead to the features of dental aerosols that we currently consider to be high risk. This has enabled us to choose drill parameters to keep our patients and the dental team safe at this difficult time. Although we cannot provide every procedure, because slowing our drills is much less efficient, we now have the basis to do more than we have done in the last six months'.

The results ${ }^{1}$ have been published in the Journal of Dental Research and are already being included as evidence in guides for dental practices in the UK during the pandemic. The collaborative research used the engineering expertise at Imperial and clinical expertise at King's College London's Faculty of Dentistry, Oral \& Craniofacial Sciences.

The researchers used dental clinical rooms at Guy's Hospital in London to test how aerosols are generated during procedures such as caries removal, applying and polishing fillings, and adjusting prostheses.
They measured the aerosol generation using high-speed cameras and lasers. They then used these findings to suggest modifications.

They found that using air turbine drill types, which are the most common type of dental drill, creates dense clouds of aerosol droplets that spread as fast as 12 metres per second and can quickly contaminate an entire treatment room. Just one millilitre of saliva from infected patients contains up to 120 million copies of the virus, each having the capacity to infect.

They tested a different type of drill, known as high torque electric micromotor, with and without the use of water and air streams. They found that using this drill type at low speeds of less than 100,000 rpm without air streams produced 60 times fewer droplets than air turbine drill types.

In addition, they found that aerosol concentration and spread within a room is dependent on the positioning of the patient, presence of ventilation systems, and the room's size and geometry. It is also influenced by the initial direction and speed of the aerosol itself, which can be affected by the type of cutting instrument (burr), and the amount and type of cooling water used.

The researchers say that, by understanding how to reduce the amount of aerosol generated in the first place, their suggestions could help dentists to practise more and help patients get the treatment they need. They also note that patients should still not attend dental appointments if they have symptoms of COVID-19.

Their suggestions have been included in the evidence appraisal in dentistry document Rapid review of aerosol generating procedures in dentistry, published by the Scottish Dental Clinical Effectiveness Programme (SDCEP). The results from the study have also been considered by an expert task force convened by the Faculty of General Dental Practice UK and the College of General Dentistry and published in their guide Implications of COVID-19 for the safe management of general dental practice.

\section{Reference}

1. Sergis A, Wade W G, Gallagher J E et al. Mechanisms of atomization from rotary dental instruments and its mitigation. J Dent Res 2020; DOI: $10.1177 / 0022034520979644$ 